\title{
BAND STRUCTURE FORMATION IN METALS UNDER DEFORMATION
}

\author{
V.V. GUBERNATOROV*, B.K. SOKOLOV, A.K. SBITNEV, \\ L.R. VLADIMIROV and I.V. GERVASYEVA \\ Institute of Metal Physics, Ural Division of Russian Academy of Sciences, \\ 18, Kovalevskaya Street, 620219 Ekaterinburg, Russia
}

(Received in final form 28 September 1997)

The study of the specific features of the flow of different metal layers at the deformation zone during rolling suggests that a regular banded structure of the material is determined by corrugation of some of its layers subject to strain.

Keywords: Rolling; Deformation zone; Corrugation; Bands

\section{INTRODUCTION}

Plastic deformation and annealing of deformed materials are the most popular methods used to effectively alter the structure and the crystallographic texture of metals and, correspondingly, their structural properties. Unfortunately, these methods cannot be realized to their full capacity because of the problems arising from the fact that up to now there has been no clear understanding of the reasons and mechanisms responsible for certain structures and textures formed in metals under strain.

In particular, to this day there has been no answer to the following question: Why and how is a regular mesoscopic banded structure deformation bands, transition bands and shear or kink bands - formed during rolling in the $\left[\begin{array}{lll}0 & 0 & 1\end{array}\right]$ direction in a sheet single crystal $\left(\begin{array}{lll}1 & 1 & 0\end{array}\right)$ of the

\footnotetext{
* Corresponding author.
} 
$\mathrm{Fe}-3 \% \mathrm{Si} \mathrm{BCC}$ alloy, which may be considered as a structurally homogeneous medium?

To determine the reasons responsible for the appearance and a regular arrangement of the banded structures, we examined the character of the metal flow during cold-rolling. The subject of study was single sheets crystal of the $\mathrm{Fe}-3 \% \mathrm{Si}$ alloy. The metal flow was determined by changes in the macro- and mesostructure at the deformation zone.

\section{EXPERIMENTAL RESULTS AND DISCUSSION}

Macroinhomogeneities - deformation twins whose orientation is close to $\left(\begin{array}{lll}1 & 0 & 0\end{array}\right)\left[\begin{array}{lll}0 & 1 & 1\end{array}\right]$ - appear in a single crystal $\left(\begin{array}{lll}1 & 1 & 0\end{array}\right)\left[\begin{array}{lll}0 & 0 & 1\end{array}\right]$ subject to rolling of $2-5 \%$ at sub-zero temperatures. They are shaped as thin plates having rectilinear boundaries, which go across the rolling direction on the surface of the single crystal. As this single crystal undergoes further rolling at room temperature, the twins are rolled and acquire a wavy shape, i.e. are corrugated (Gubernatorov and Sokolov, 1972).

The main reason for the corrugation of the twins is deemed to be as follows. During rolling of a twinned single crystal the matrix and the twins are deformed differently by their crystallography: the twins are expanded to a greater extent than the matrix. As a result of different expansions of the matrix and the twins, the latter are deformed under constrained conditions (the matrix obstructs the expansion of the twins) and they have to corrugate.

Thus, it may be inferred that if the metal flow is hampered (obstructed) in some layer during deformation, compression stresses arise in this layer. When these compression stresses exceed the critical stress, the layer becomes unstable and is corrugated.

The corrugation of the twins predetermined the line of our further investigations. We examined the flow of different metal layers at the deformation zone in order to ascertain the presence of metal layers, which have to corrugate during rolling.

An unconventional examination of the reduction of the vertical layers of a sample at a small (elementary) pass between the rolls (the roll spacing being constant) revealed the following. In accordance with its geometry, the deformation zone proved to have a vertical metal 
layer, which undergoes the maximum instantaneous absolute reduction ( $\left.h_{i}=h_{i}-h_{i+1}\right)$ and, correspondingly, is expanded most (Fig. 1).

Quite probably it is this layer that will be corrugated. The corrugation will take place over the width and thickness of the sample and along the rolling direction. The corrugation of a metal layer on the width of the sample is caused by the fact that this layer widens under constrained conditions like a twin.

The layer will be corrugated over the sample's thickness, because it experiences a vertical compression and therefore the core system becomes unstable. Finally, among all the layers, the layer which undergoes the maximum reduction flows most in the horizontal direction (along the rolling direction). However, the adjacent layers (rigid edges) obstruct the expansion of this layer and make it corrugate.

It may be assumed that the corrugation of the layer along the rolling direction is responsible for the appearance of shear (kink) bands, while the corrugation of the layer over the width and thickness of the sample curves the shear (kink) bands and assigns the crystal re-orientation direction, that is, stimulates the formation of deformation bands. This supposition is confirmed by the fact that the surface mesostructure of the single crystals $\left(\begin{array}{lll}1 & 1 & 0\end{array}\right)\left[\begin{array}{lll}1 & 1 & 0\end{array}\right]$ and $\left(\begin{array}{lll}1 & 1 & 0\end{array}\right)\left[\begin{array}{lll}0 & 0 & 1\end{array}\right]$ change along the length

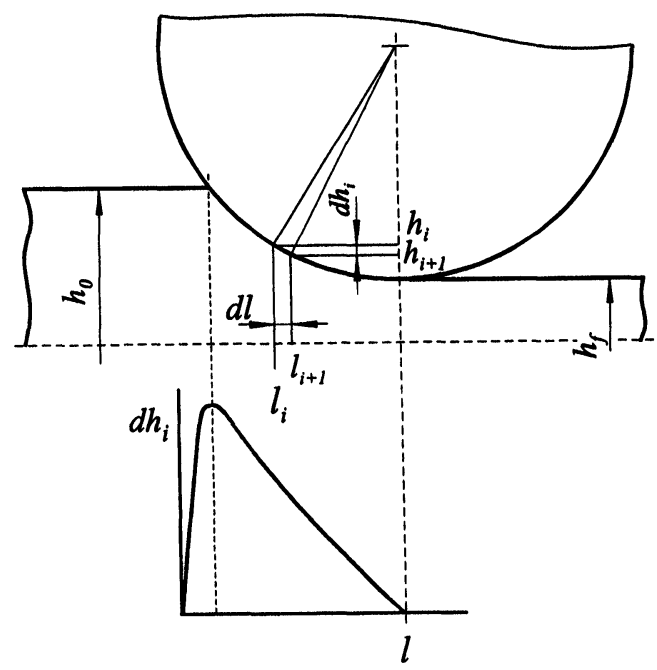

FIGURE 1 Scheme of deformation zone and the distribution of instantaneous absolute reduction. 


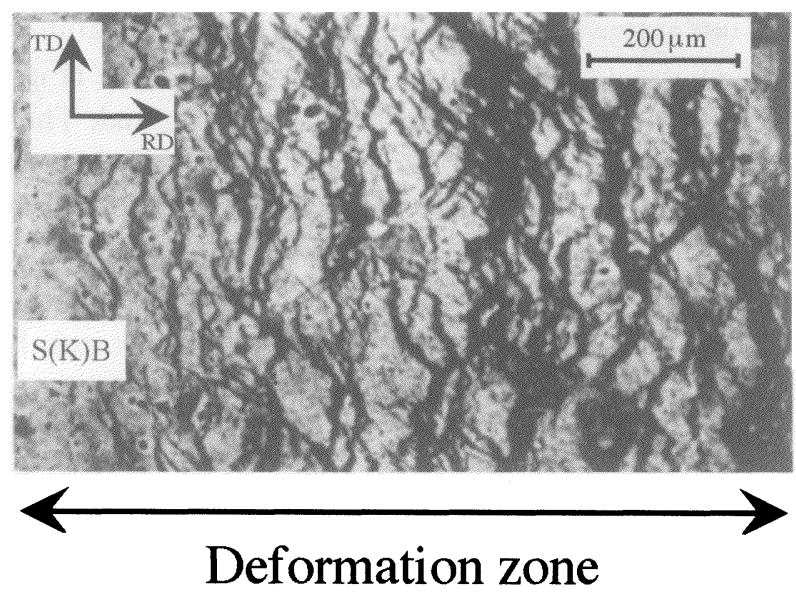

FIGURE 2 Mesostructure of rolling surface for single crystal (1 10$)\left[\begin{array}{ll}1 & 1\end{array}\right]$ along the deformation zone; $\mathrm{S}(\mathrm{K}) \mathrm{B}$ - shear (kink) bands.
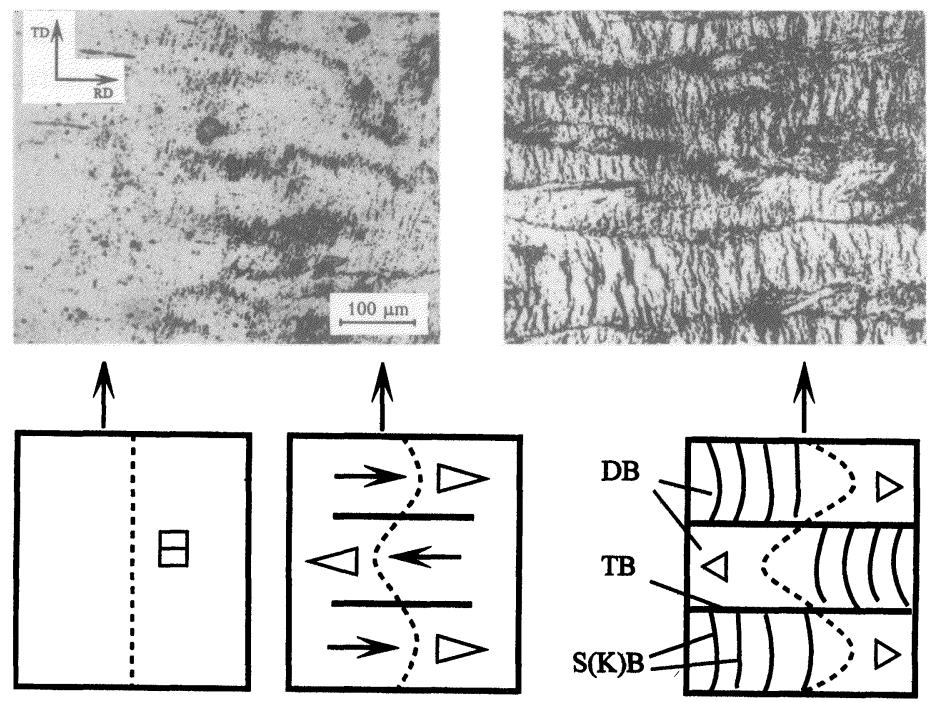

\section{Deformation zone}

FIGURE 3 Mesostructure of rolling surface for single crystal (1 110$)\left[\begin{array}{lll}0 & 0 & 1\end{array}\right]$ along the deformation zone and the scheme for deformation bands and shear (kink) bands

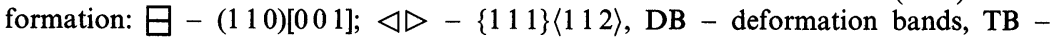
transition bands. 
of the deformation zone (l), Figs. 2 and 3. From Fig. 3 it is seen that shear (kink) bands appear within the deformation bands formed. They are due to the metal corrugation, which results from different mechanical properties of adjacent deformation bands.

Moreover, one should allow for possible corrugation of the layers in the longitudinal cross section of the samples. This corrugation is related to different degrees of deformation in the cross-section. A distinction of deformation behaviour is determined by the ratio between the length of the deformation zone, $l$, and the mean cross-sectional thickness, $h_{\mathrm{m}}$, of the rolled sample, $h_{\mathrm{m}}=\left(h_{0}-h_{\mathrm{f}}\right) / h_{0}$, where $h_{0}$ is the initial thickness, and $h_{\mathrm{f}}$ the final thickness. When $l / h_{\mathrm{m}}>(0.5-1.0)$, surface layers corrugate. When $l / h_{\mathrm{m}}<(0.5-1.0)$, internal layers of the metal corrugate.

\section{CONCLUSION}

The banded structure of a rolled metal is determined by the corrugation of some metal layers at the deformation zone. This corrugation is explained by the specific features of the metal flow in various directions. The rolling parameters affect the corrugation characteristics and the morphology of the banded structures formed. Thus, the possibility opens up to purposefully control the structural and texture characteristics of deformed and recrystallized metals.

The corrugation of the metal layers at the deformation zone and a significant role played by the corrugation in the appearance of the deformation structure and texture bear a universal character. The corrugation occurs at other types of deformation (for example, in tension of flat bars and during fatigue tests) and during phase transformations when the specific volume of the new phase is larger than that of the initial phase.

\section{References}

Gubernatorov, V.V. and Sokolov, B.K. (1972). Influence of twins on the deformation and recrystallization texture in single crystal of $\mathrm{Fe}-3 \% \mathrm{Si}$ alloy. Fizika Metall. $i$ Metalloved. 34(6), 1232-1237. 